\title{
Homotypic Non-exclusion by Vesicular Stomatitis Virus in Chick Gell Culture
}

\author{
By P. D. COOPER* \\ Wright-Fleming Institute of Microbiology, St Mary's Hospital Medical School, \\ London, $W .2$
}

SUMMARY: Placing live or inactivated vesicular stomatitis virus of one serotype on chick cells in tissue culture prevented most of the cells from releasing infective virus of the other serotype when super-infected with it (heterotypic exclusion). Inactivated virus did not prevent super-infection with the same serotype and had no effect on the latent period or rate of virus release (homotypic non-exclusion and non-interference). The 'Indiana' serotype was more effective as heterotypic excluding agent than was the 'New Jersey' serotype, and exclusion was noticeable when only 12 min. elapsed between interfering and challenge virus. Each cell liberated virus of only one serotype when infected with live virus of both serotypes, but the serotype released was often $(20-40 \%)$ not that of the particle first adsorbed. Heterotypic exclusion in fact behaved as if it were reversible and dependent on the multiplicities of infection, at least within the latent period. Many inactivated particles per cell were adsorbed before heterotypic exclusion was achieved.

The phenomenon of 'shortened latency' in vesicular stomatitis (VS) virus infections of chick embryo cells (Cooper, 1958), where a higher multiplicity of infection caused the first infective progeny to appear sooner, raised the question of whether one particle of this virus was able to exclude an identical particle from infecting the same cell. Clearly any interpretation of shortened latency is affected by exclusion, since then all adsorbing virus would not be contributing equally to the progeny.

This paper demonstrates the presence of heterotypic exclusion by live or inactivated preparations of VS virus (Indiana or New Jersey serotypes) under conditions where the same inactivated preparations do not exclude the live homotypic virus. The conclusion is drawn that early exclusion between live homotypic particles is therefore also unlikely, although not disproven.

Some interpretations of shortened latency are discussed elsewhere (Cooper, 1958); because of the particular relevance to shortened latency the present paper examines interference mostly in the early latent period.

\section{METHODS}

Virus stocks were high titre dilute-passage stocks containing minimal amounts of the non-infective interfering component (Cooper \& Bellett, 1958), and were prepared by growth on chick embryo cell monolayers as described by Cooper (1957). Virus titres were obtained by plaque assays on chick embryo mono-

* Present address: Virus Culture Laboratory, Medical Research Council Laboratories, Carshalton, Surrey. 
layers as described in the latter paper. The guinea-pig hyper-immune antisera used in separating the serotypes (kindly given by the Research Institute, Pirbright) had no effect on plaque counts of their respective serotypes when incorporated to a concentration of $1 / 6000(\mathrm{v} / \mathrm{v})$ in the agar overlay, but completely prevented plaque formation at $1 / 600$; a concentration of $1 / 120$ was routinely used, and its effectiveness usually checked for each experiment. Plaque counts with heterotypic antisera of either type were completely unaffected at $1 / 60(\mathrm{v} / \mathrm{v})$.

Ultraviolet inactivation was accomplished by irradiating 1 or $2 \mathrm{ml}$. of tissue culture fluid for $1-5 \mathrm{~min}$. in a $10 \mathrm{~cm}$. Petri dish at $18^{\circ}$ on an automatic rocking device which tilted the dish once every 2 sec. Under these circumstances one lethal hit occurred about once every $40 \mathrm{sec}$. The dose for distilled water was about 5 ergs mm. ${ }^{-2}$ sec. $^{-1}$.

\section{Technique for establishing conditions of exclusion}

All experiments were made by adding a primary inoculum to $20 \mathrm{hr}$. chick embryo monolayers (usually the interfering agent) followed after a period of adsorption by removal of the inoculum, addition of the secondary inoculum (usually the challenge virus) and its removal after a second period of adsorption. These are described where appropriate. Usually cells were washed and removed by trypsinization for assay of infective centres as described below; exclusion is therefore defined and used in the bacteriophage rather than the sperm-ovum sense, i.e. a prevention of a potentially adsorbing and infecting virus from liberating infective progeny characteristic of its own serotype, and therefore from forming a plaque neutralized by its own antiserum.

All operations up to cell chilling were made in an incubator hood, which is a Perspex box with sloping front and sleeved arm-holes designed for manipulating Petri dish monolayers in an atmosphere of controlled temperature, $\mathrm{CO}_{2}$ content and humidity. Thus temperature, $\mathrm{pH}$ and tonicity variations in the monolayers were eliminated during the early latent period, which was both the time under investigation and the time potentially affected by the manipulations. The similarity of one-step growth curves produced in the incubator hood with those produced without it showed that such variations are not important for growth curves provided that they are soon corrected (usually within 15 min.); their elimination was important for the present purpose as otherwise they might be incriminated as potential causes for the lack of homotypic exclusion described below.

Harvesting cells for assay. After the final adsorption of seed, fluid was aspirated and drained as completely as possible, plates were washed twice with PBS (phosphate buffered saline, Dulbecco \& Vogt, 1954), and cells removed by $5 \mathrm{~min}$. treatment with $5 \mathrm{ml}$. trypsin $\left(2.5 \mathrm{mg} . / \mathrm{ml}\right.$. in PBS) at $37^{\circ}$. The suspension was thoroughly chilled in ice, centrifuged, washed once in PBS and suspended in $1 \mathrm{ml}$. cold $50 \%(\mathrm{v} / \mathrm{v})$ PBS in 'conditioned' medium (centrifuged medium from formed $20 \mathrm{hr}$. monolayers). After $5 \mathrm{~min}$. standing to settle large clumps, the supernatant $0.75 \mathrm{ml}$. was removed for assay of infective centres and total cells (haemocytometer count). The suspension was 
then almost entirely monodisperse. Cells were plated while still in the early latent period; after all plating of cells was complete, free virus was estimated as the infective centres remaining after $3 \mathrm{~min}$. centrifuging at $1000 \mathrm{rev} . / \mathrm{min}$. Some estimation of the higher limit of 'associated' virus, i.e. externally attached plus internally contained mature virus, was obtained as the number of infective centres remaining after one cycle of freezing at $-70^{\circ}$ and thawing; the relevance of this control is that the externally attached virus may elute and give spuriously high infective centre counts. Such elutable and free virus was always low compared with the total infected cell count. It is felt that the bulk of the 'associated' virus in the present work is accounted for by the undisrupted cells still present (up to $10 \%$ of the total).

\section{RESULTS}

\section{Heterotypic but not homotypic exclusion by inactivated VS virus}

Table 1 shows that when monolayers of chick cells from the same batch were allowed to adsorb a high multiplicity of 'storage' $-\left(-20^{\circ}\right)$ plus ultravioletinactivated (UVI) virus particles of either New Jersey (NJ) or Indiana (IND) serotype, $100 \%$ of the cells thus infected could produce progeny from a single homologous active particle added subsequently, but only $20 \%$ (IND VS as primary inoculum) or $40 \%$ (NJ VS as primary inoculum) from a single subsequent heterologous particle. This lower efficiency of NJ VS as an interfering agent was evident in all of three experiments where it might be found, despite a frequently higher multiplicity of infection by the NJ VS.

In these experiments the interfering agent was given the greatest opportunity of establishing itself, consistent with limitation to the early part of the latent period, namely, adsorption for $1 \mathrm{hr}$., frequent respreading of the inoculum, and high multiplicity of infection. It can be seen that, despite this maximum opportunity, the heterotypic exclusion is not efficient in the sense that by no means all the cells having the interfering agent are prevented from producing progeny. The primary inoculation was carried out by a method which had been shown with live virus to infect nearly all of the cells (Cooper, $1955,1957)$, so that all cells are likely to receive inactivated virus. Nevertheless, although heterotypic exclusion is not complete, its presence in $60-80 \%$ of the cells rules out the possibility that the non-appearance of homologous exclusion in the same experiment was due to some failure of the majority of cells to be infected by the primary inoculum, for example, that the inactivated virus did not adsorb.

Since these preparations are certain to contain much heat-inactivated virus (the half-life of VS at $37^{\circ}$ is $1-3 \mathrm{hr}$. in the usual growth media, Cooper \& Bellett, to be published), the lack of homotypic exclusion applies to heatinactivated as well as to UVI and storage-inactivated virus.

The heterotypic exclusion by inactivated NJ or IND strains was reciprocal, but whether 'mutual exclusion' occurs in the sense that only one but not both serotypes can grow together in the same cell will be discussed below. As was found repeatedly in other experiments, no multiplicity reactivation was 


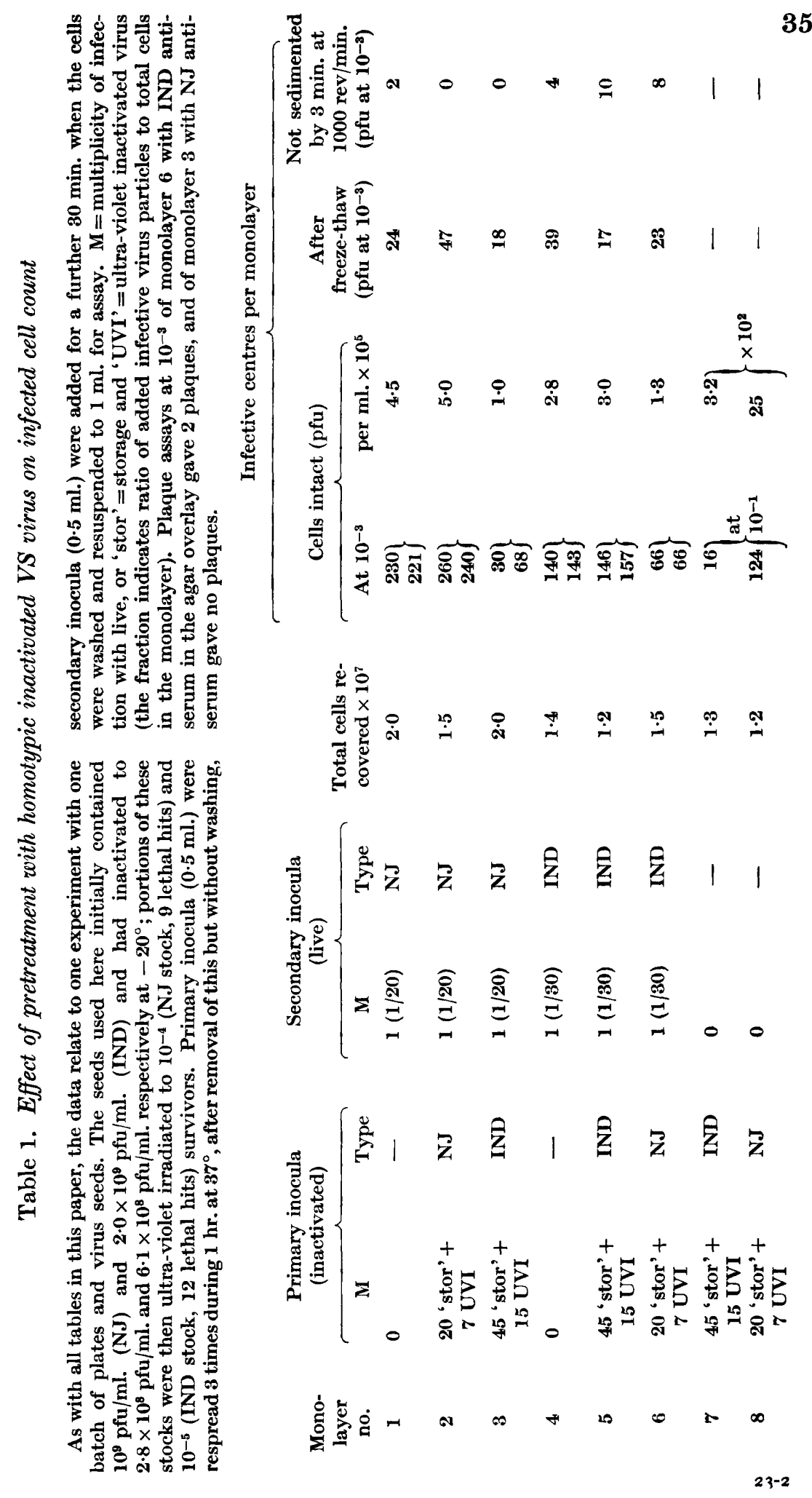


evident in Table 1 among UVI or storage-inactivated particles, as the plating efficiency of the cells receiving inactivated virus (monolayers 7 and 8 ) in terms of the ratio of cells infected (pfu added): infective centres recovered was the same as the controls (monolayers 1 and 4). No reactivation of the inactivated serotype by the active was detected either (monolayers 3 and 6 , columns 9 and 10), in contrast to the findings of Gotlieb \& Hirst (1956) with influenza; this may be due to the lack of relationship between the two serotypes found serologically (Brooksby, 1949).

Table 2 shows that homotypic UVI virus still had no excluding action when added up to $\mathbf{3 0} \mathrm{min}$. after the active virus.

\section{Heterotypic exclusion by live virus}

It does not seem possible to show exclusion by live virus directly for any preparation where the inactivated particles exclude and where a content of less than $\mathbf{5 0} \%$ inactive particles cannot be guaranteed. It is possible that all the reciprocal heterotypic exclusion found in the present work by preparations containing live virus is due to the inactive particles also contained. The most that can be said at present is that wholly inactive preparations behave similarly to preparations containing not more than $50 \%$ active particles, the rest being heat- or storage-inactivated (Table 3). As in Table 1, exclusion is reciprocal between NJ and IND, but IND is the more efficient excluding agent. Free and associated infective virus was negligible.

\section{Minimum time to establish exclusion}

Table 3 shows that with NJ VS as the primary inoculum and IND as the secondary, addition of the secondary inoculum only $12 \mathrm{~min}$. after the primary still caused a marked decrease in the number of cells releasing IND VS. The significance of this is discussed below.

\section{Likelihood that heterotypic exclusion is reversible}

Heterotypic exclusion is not complete: $20-40 \%$ of the cells infected by the primary serotype yield virus of the secondary serotype. This indicates either that some cells can support growth of both serotypes at once, or that the process of infection is reversible in many cells at the stage at which they have been challenged.

Table 4 shows the results of infecting cells with both NJ and IND serotypes in such a way that the efficiencies of exclusion were about equal, i.e. the less efficient NJ serotype was present in threefold excess of multiplicity. Significant elution of cell-associated virus from infected cells has never been found in these experiments. The only interpretation of the experiment of Table 4 is, therefore, that the secondary serotype overcame the primary in almost precisely half the cells, while mixed yielders were negligible. Mutual exclusion was demonstrated: a cell was able to liberate VS virus of only one serotype, but the primary infection was reversed in half of the population by the secondary serotype. This was so whether the primary serotype was IND or NJ. 


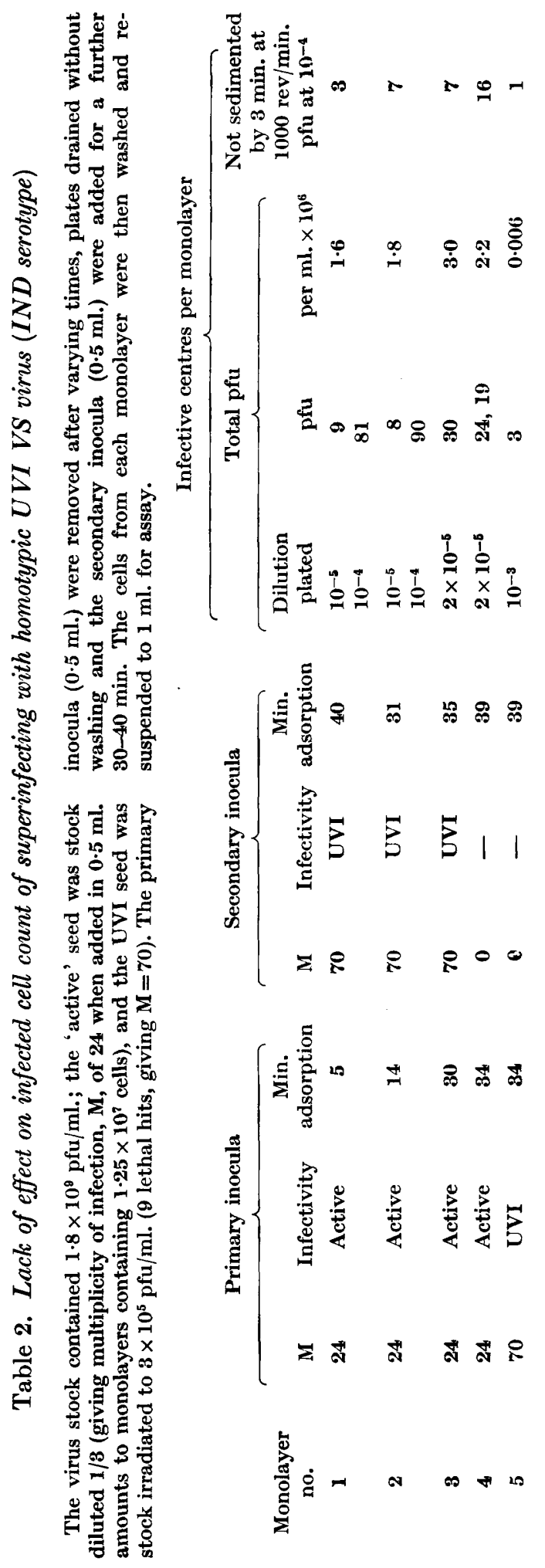




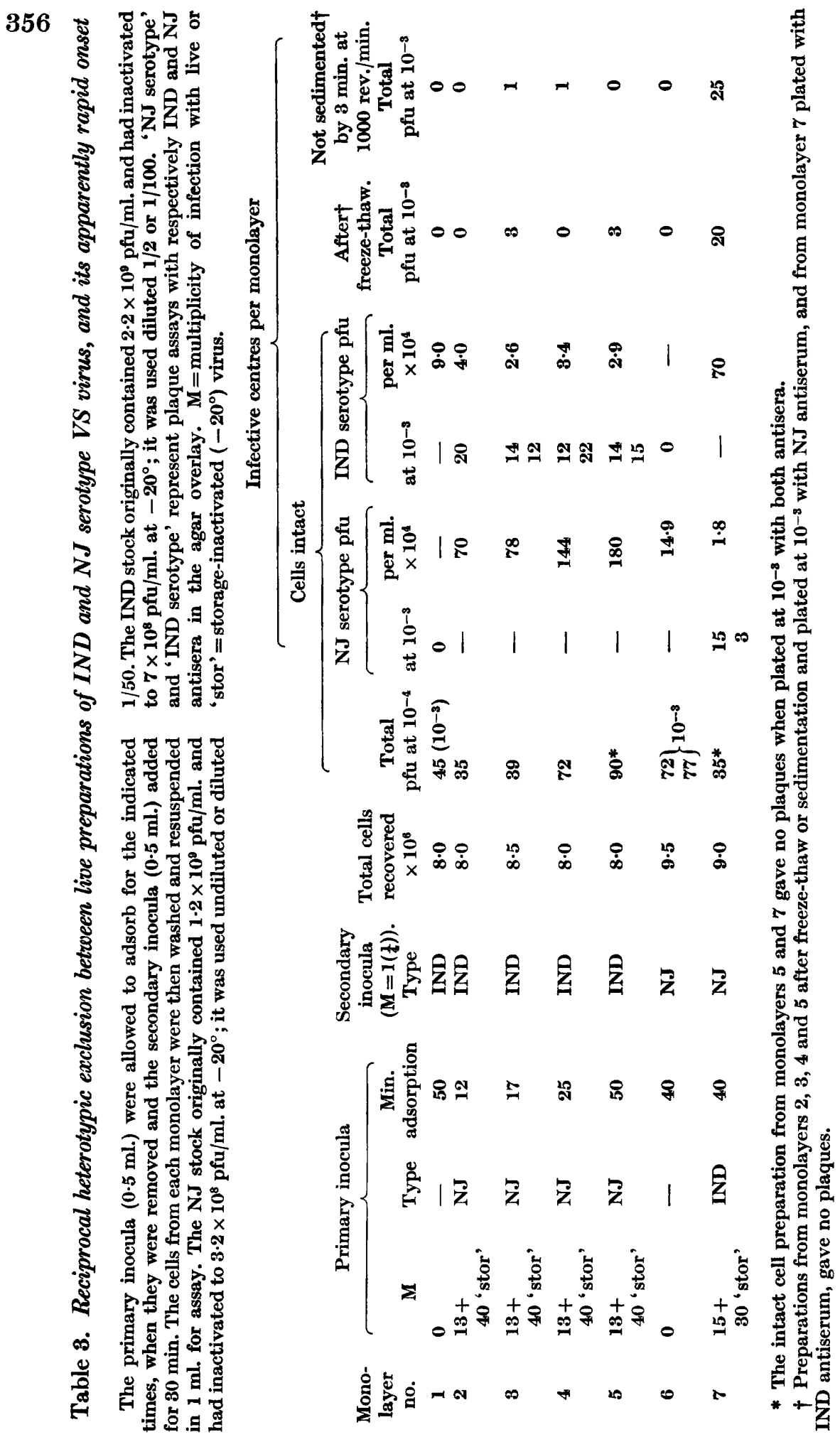




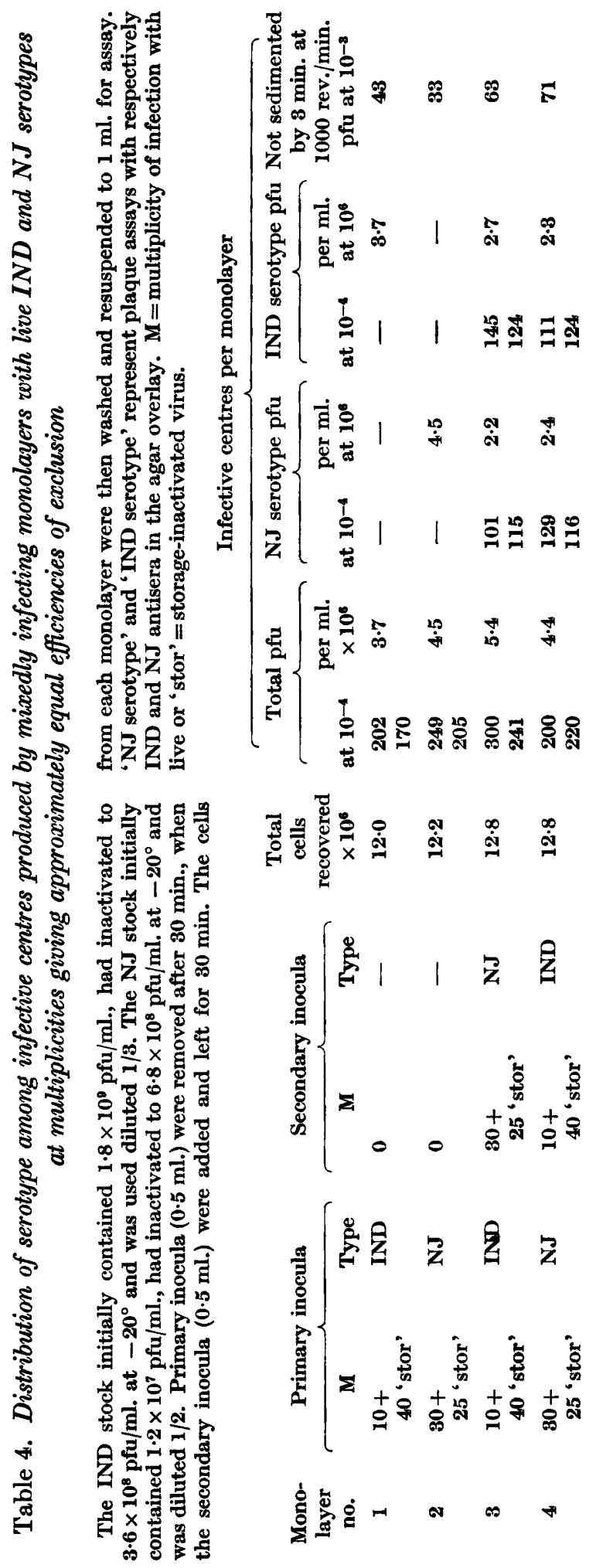


Table 5 shows that the efficiency of reversal (proportion of secondary to primary serotypes in the total yield of infective centres) was considerably increased when the multiplicity of infection of the secondary serotype was increased. Free and elutable virus was negligible.

\section{Minimum number of particles required for heterotypic exclusion}

As the total number of particles (infective plus non-infective) in the VS preparations is not known, and as inactive particles can be shown to exclude heterotypically, any estimate of number of particles required for exclusion must be regarded as a minimum. Table 6 shows that, after primary adsorption of inactivated NJ VS to varying multiplicity, the number of secondary serotype infective centres present in a monolayer after secondary adsorption of about 80 live heterotypic particles ( 1 per $2 \times 10^{5}$ cells) was significantly affected only when the average multiplicity of primary infection was between 1 and 5 .

Comparison of these data with those of Baluda (1957), who showed that only one inactivated Newcastle disease virus particle was required to initiate exclusion, strongly suggests that many VS particles are required. This conclusion is supported by the large number of yielders (20-40\% of total cells) usually found in cell populations completely infected with a high multiplicity of active or inactive interfering virus, despite a low (single) multiplicity of challenge virus. Since much heat-inactivated virus must also be present the process does not seem very efficient, but adsorption of the higher number of primary particles in Table 6 improved the efficiency, due perhaps to cooperation between particles, or an increased probability that a single particle may succeed.

Plaque size was also markedly reduced at about the inoculum size where most cells had received one inactivated particle, but it is noteworthy that plaques were still able to form when the multiplicity was 50. On these high multiplicity plates the cells between plaques stained well with neutral red and did not differ from controls in microscopical appearance. Thus inactivated virus alone did not harm cells, but in the plaques all cells were unstained and showed much cytopathic effect, so that the initial protection once afforded to at least half the cells had lapsed, either by the passage of time or by reversal by a high multiplicity of infection within the plaque. On the other hand, the fact that plaques were smaller means that interference must in many cells and in some form have lasted at least as long as the time required for infection of the second cycle in the plaques (4-12 hr.).

\section{Lack of effect of pre-inoculation with inactivated homotypic VS virus on release curves}

Two monolayers were inoculated with a high multiplicity of UVI + storageinactivated IND VS and a third with medium only as a control. After adsorption, one inactivated-inoculated plate and the control plate were completely infected with a low multiplicity (average $=2$ ) of active homotypic virus, and the other inactivated-inoculated plate had medium only as a second control. After a further adsorption period, the cells were removed with trypsin and 


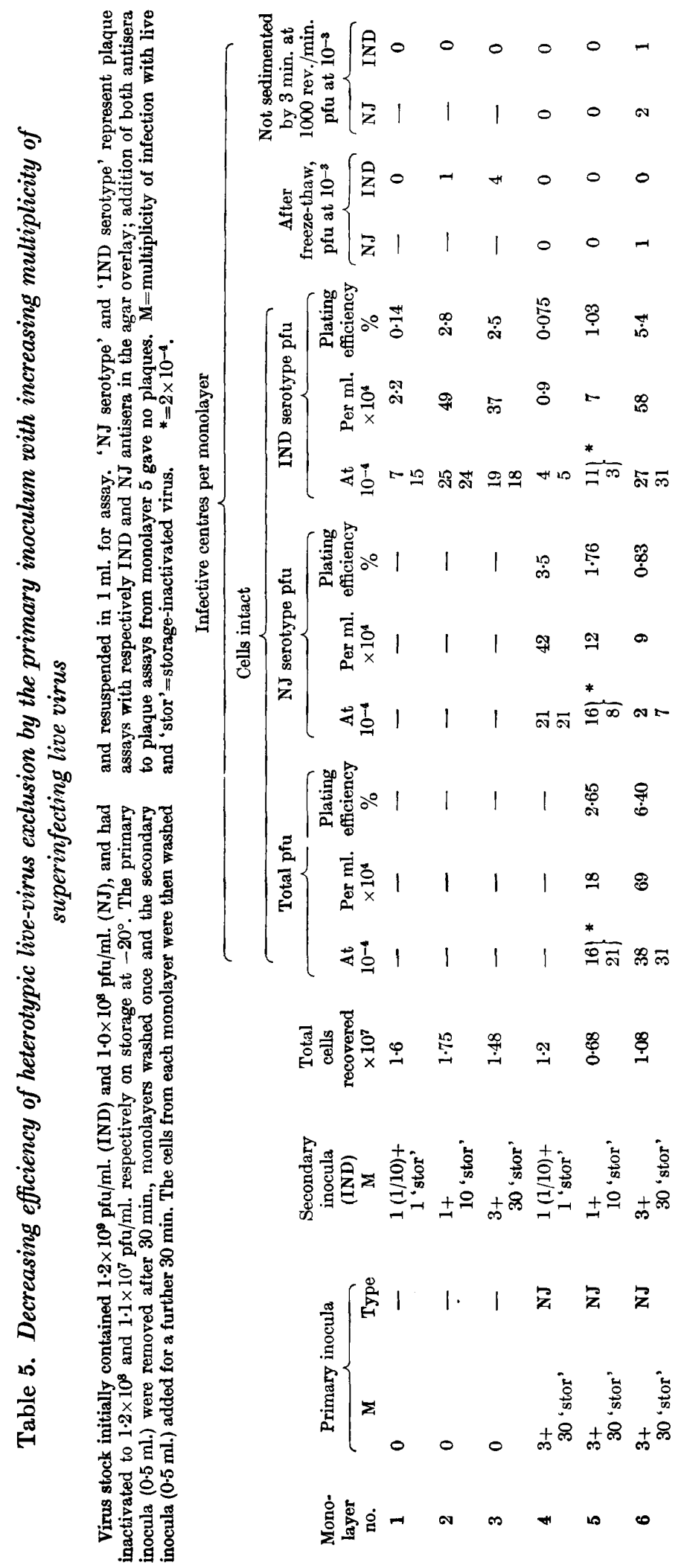


360

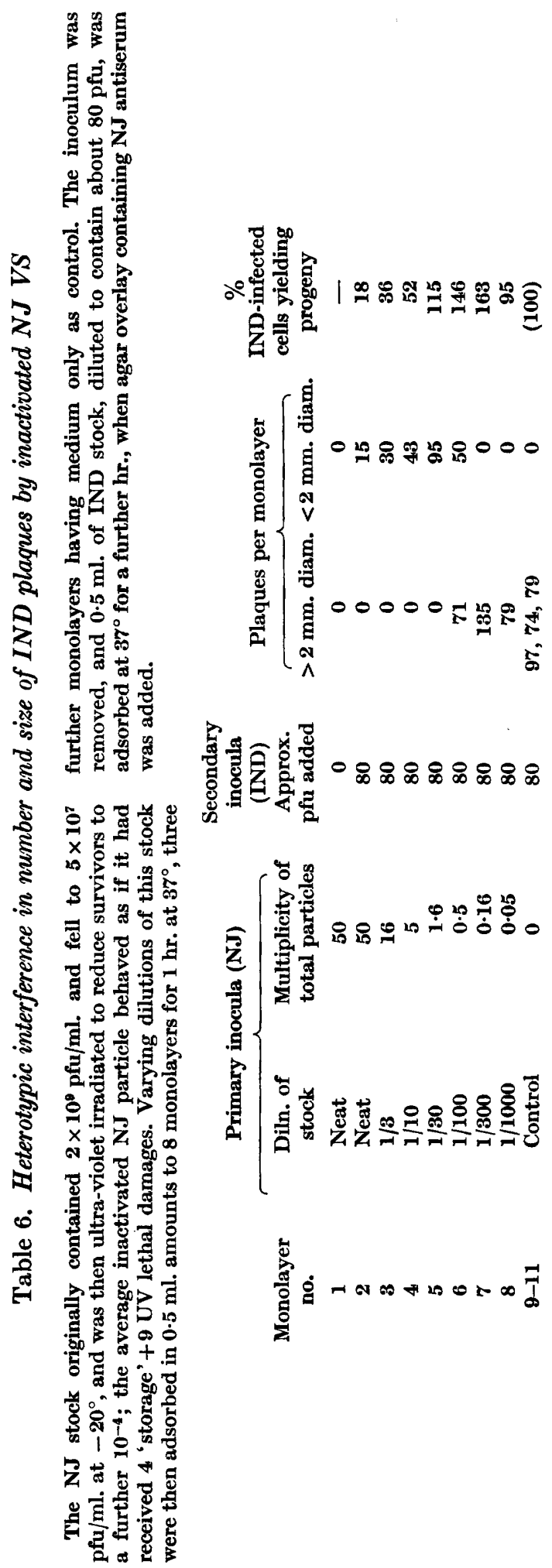




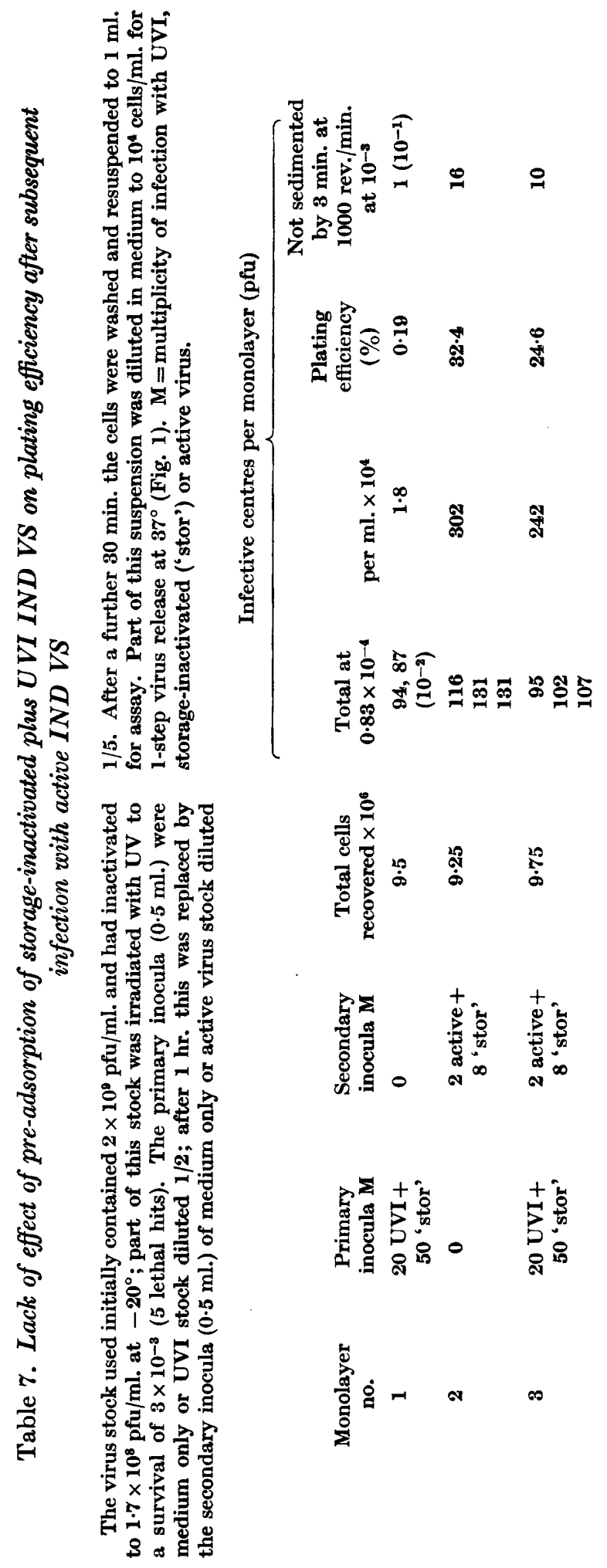


suspended to $10^{4} / \mathrm{ml}$. in a $50 \%(\mathrm{v} / \mathrm{v})$ mixture of PBS and 'conditioned' medium for one-step virus release at $37^{\circ}$. Figure 1 . shows that this preadsorption of inactivated VS had no effect on the release rate or latent period; a slight apparent decrease in final yield was not confirmed in other experiments and is not regarded as significant. As in Table 1 there was also no significant exclusion in terms of number of infective centres recovered (Table 7 ). In the suspension inoculated with inactivated virus only, the ratio of infective centres

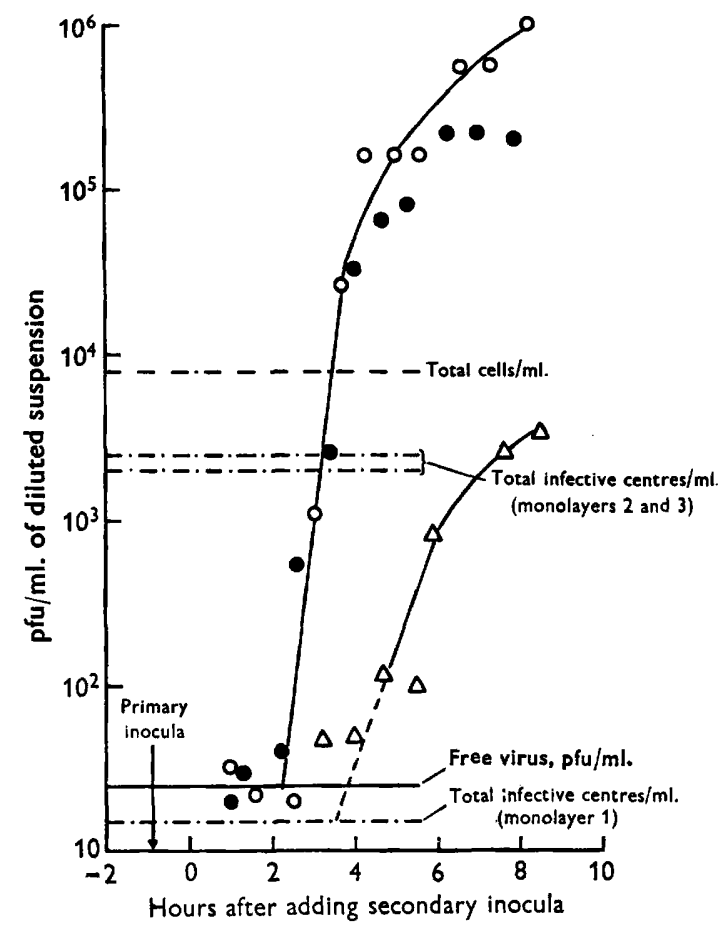

Fig. 1. Lack of effect of pre-adsorption of storage-inactivated plus UVI IND VS on 1-step virus release in suspension at $37^{\circ}$ after subsequent infection with active IND VS. The data are from the same experiment as those of Table 7. $\triangle$, monolayer 1 ; $O$, monolayer 2; 0 , monolayer 3.

recovered to active particles added was if anything slightly less than the plating efficiency of the other cells, $13 \%$ compared with $30 \%$, indicating that multiplicity reactivation was not occurring. The release curve of this suspension is less clear in the early stages than the others, but the latent period (3-5 hr.) is not very different from that usual for single infection (4.5 hr.) and the rate of release and final yield are also not much affected, despite the large excess of inactive virus adsorbed within the same $1 \mathrm{hr}$. period.

\section{DISCUSSION}

A problem raised by the phenomenon of shortened latency (Cooper, 1958), and the one dealt with in this paper, is whether or not each adsorbing particle of VS virus during more or less simultaneous multiple infection (i.e. during the 
first half-hour of the latent period), contributes equally to the synthesis of progeny.

Interference and exclusion occur widely among viruses, and the precedent from bacteriophage work suggests that mutual exclusion may occur between unrelated viruses, but related viruses may allow some mixed infection. On the other hand, interference showing as cross-protection is a criterion of relationship among plant viruses. One cannot, therefore, predict from these cases whether exclusion might occur between virus particles of the same or different serotypes of VS virus.

One practical criterion for the problem stated above is the absence or presence of exclusion in the sense of the non-release, in infective form, of a particular genome participating in a mixed infection. Homotypic interference in the sense of depressed rate of release and lower yield by live virus (compared at varying multiplicities of infection) has been shown not to occur with dilute passage stocks of VS virus (Cooper, 1958), although undiluted passage stocks of both VS serotypes contain a non-infective component capable of marked homotypic exclusion (Cooper \& Bellett, to be published). The present paper shows that UVI, heat- and 'storage'-inactivated dilute-passage VS virus preparations do not exclude live virus homotypically under conditions where the same inactivated preparations markedly exclude heterotypically. Live virus preparations also exclude heterotypically. The many observations (Luria, 1953) that practically no interfering phenomenon shown by live virus is destroyed by a relatively low number of UV lethal hits makes it seem likely that live VS virus will not exclude homotypically, so that all particles of a mixed infection should contribute equally to progeny. A more definite conclusion for VS virus must await further evidence, perhaps from genetically labelled strains.

Among the many cases of interference by animal viruses, the easiest interpretations, from a cellular viewpoint, involve influenza and Newcastle disease virus (NDV). Like VS, inactivated influenza virus interferes completely and rapidly with heterologous virus (except that only 1 particle may be required per cell (Fazekas de St Groth \& Edney, 1952)), but not with homologous virus (save for some depression of yield), provided that super-infection occurs within a few hours (Frazer, 1953). On the other hand, Baluda (1957) showed that UVI NDV excluded homotypically and rapidly, and evidence was given to suggest that the content of non-infectious haemagglutinin was low. However, where 'incomplete' interfering virus may exist and where homotypic exclusion has been found in inactivated virus preparations (unlike dilutepassage VS), further evidence is required to ensure that such exclusion cannot be due to a relatively low content of non-infectious haemagglutinin, e.g. equal in number to infective particles. Baluda also found that, like heterotypic VS, the exclusion in homotypic NDV was to some extent reversed by an increase in multiplicity of the superinfecting virus, although the exclusion appeared much more efficient in the case of NDV in that one particle only was required, whereas VS (heterotypic) probably needs several or very many. VS (homotypic) is even less efficient in that no exclusion was detectable up to 
multiplicities of 70; this contrasts markedly with the efficiency of the non-infectious component of VS undiluted passage (Cooper \& Bellett, to be published), in which a small number of particles, perhaps one, excluded homotypically.

The presence of marked heterotypic exclusion in VS when the secondary inoculum was added only $12 \mathrm{~min}$. after the primary suggests that the process of establishing exclusion may be quite rapid. It should be made clear, however, that this may not be the case. If the apparent critical time for exclusion of the secondary particle was, for example, $1 \mathrm{~min}$. after the primary, this merely means that the process for irrevocably establishing the secondary infection is shorter by one minute than the process of irrevocably establishing exclusion. Only in the special case where the time for completely establishing the secondary infection is infinitely small can exclusion be said to be established in $1 \mathrm{~min}$. The process could in fact be quite lengthy, and an apparently rapid exclusion indicates rather that the two processes of infection and exclusion are of similar length. The apparent reversibility of exclusion with increasing multiplicity of secondary infection (which may also explain the minority of cells which fail to show heterotypic exclusion) may be explicable in terms of a competition for a particular site or of a change akin to shortened latency in probability or rate of establishment either of infection or of exclusion which is due to the increase in multiplicity of infection.

I am grateful to the staff of the Research Institute, Pirbright, Surrey, for the original virus stocks, and for several generous gifts of high titre specific antisera.

\section{REFERENCES}

BaludA, M. A. (1957). Homologous interference by ultraviolet-inactivated Newcastle disease virus. Virology, $4,72$.

Brookssy, J. B. (1949). Differential diagnosis of vesicular stomatitis and foot and mouth disease. Examination of virus samples from Mexico with special reference to complement fixation. J. Hyg., Camb., 47, 384.

Cooper, P. D. (1955). A method for producing plaques in agar suspensions of animal cells. Virology, 1, 397.

Cooper, P. D. (1957). Some characteristics of vesicular stomatitis virus growthcurves in tissue culture. J. gen. Microbiol. 17, 327.

Cooper, P. D. (1958). 'Shortened latency' as a result of multiple infection by vesicular stomatitis virus in chick cell culture. J. gen. Microbiol. 19, $\mathbf{3 4 0 .}$

Dulbecco, R. \& Vogt, M. (1954). Plaque formation and isolation of pure lines with poliomyelitis viruses. J. exp. Med. 99, 167 .

Fazeras de St Groth, S. \& Edney, M. (1952). Quantitative aspects of influenza virus multiplication. II. Heterologous interference. J. Immunol. 69, 160.

Frazer, K. B. (1953). Genetic interaction and interference between the MEL and NWS strains of influenza A virus. Brit. J. exp. Path. 34, 319.

Gotureb, T. \& HIRST, G. K. (1956). The experimental production of combination forms of virus VI. Reactivation of influenza viruses after inactivation by ultraviolet light. Virology, 2, 235.

LuriA, S. E. (1953). General Virology, p. 284. New York: Wiley and Sons. 